\title{
RESOLUÇÃO DE PROBLEMAS E GESTÃO DE PROJETOS: PROPOSIÇÃO DE OTIMIZAÇÃO PARA O SETOR METALMECÂNICO PAULISTA
}

\section{PROBLEM SOLVING AND PROJECT MANAGEMENT: OPTIMIZATION PROPOSITION FOR METALMECHANICAL SECTOR OF SÃO PAULO REGION}

\author{
André Belasques Ruiz Picon ${ }^{1}, \&$ Manoel Goncales Filho ${ }^{{ }^{*}}$ \\ 12 Universidade de São Paulo, Unidade Escola Superior de Agricultura Luiz de Queiroz \\ ${ }^{1}$ andrebpicon@yahoo.com.br ${ }^{2}$ manoel.goncales01@ fatec.sp.gov.br
}

\section{ARTIGO INFO.}

\section{Recebido em: 22.06.2021}

Aprovado em: 24.08.2021

Disponibilizado em: 14.10.2021

\section{Palavras-chave:}

Gestão de Projetos; Metodologias de Resolução de Problemas; Lean Manufacturing.

\section{KEYWORDS:}

Project Management; Problem solving; Lean Manufacturing.

*Autor Correspondente: Gonçales, M., Filho.

\section{RESUMO}

Problemas acontecem diariamente dentro de organizações e no dia-a-dia de gestores de projetos, e as formas de tratar esses problemas nem sempre são feitas considerando metodologias sistemáticas, bem estruturadas e de gerenciamento de projetos. $\mathrm{O}$ objetivo deste trabalho foi identificar na literatura as principais metodologias de resolução de problemas, coletar informações no setor industrial metalmecânico paulista sobre como são administrados e propor uma abordagem sob à ótica de gestão de projetos. O método de estudo possui abordagem quantitativa, e o procedimento técnico para o levantamento de dados foi realizado por meio de um questionário utilizando escala Likert. Os dados coletados permitiram uma análise de como os problemas são tratados no universo de estudo e demonstraram uma necessidade de melhores ferramentas para planejar como executar as atividades. Não obstante, a real e principal contribuição desta pesquisa é a proposição de implementação de uma abordagem baseada na filosofia "Lean Manufacturing" com utilização de "canvas", como formas de otimização do processo produtivo industrial para a resolução de problemas de modo estruturado.

\begin{abstract}
Problems happen daily within organizations and in the daily life of project managers. The way to deal with these problems is not always done considering systematic, well-structured and project management methodologies. However, the objective of this research was to identify in the literature the main problem-solving methodologies, collect information in the São Paulo metal-mechanical sector about how they are managed and to propose an approach from the perspective of project management. The method has a quantitative approach and the technical procedure for data collection was carried out using a questionnaire using a Likert scale. The data collected allowed an analysis of how problems are handled by the focus universe of study and they demonstrated a need for better tools to plan how to carry out activities. Nevertheless, the real and main contribution of this research is the proposal to implement an approach based on the "Lean Manufacturing” philosophy using "canvas" as ways to optimize the industrial production process to solve problems in a structured way.
\end{abstract}



o setor metalmecânico paulista. Brazilian Journal of Production Engineering, 7(5), 30-47 .

\section{INTRODUÇÃo}

Diariamente, os gestores de projetos e as empresas enfrentam diferentes tipos de problemas. Eles podem ser muito simples e serem solucionado em segundos, mas também podem ser complexos e durar por gerações. As etapas de gerenciamento de projetos incluem planejamento, organização, supervisão e controle, para alcançar um objetivo em determinado tempo (Carvalho, 2018), logo problemas podem ser tratados como projetos e utilizar essa estrutura de gestão. Segundo o IMPA (2012), a "National Competence Baseline" [NBC] define que uma das competências técnicas para um gestor de projeto é a resolução de problemas e que procedimentos sistemáticos devem ser adotados para buscar uma solução rápida e eficiente.

O Controle de Qualidade Total (CQT), tema relacionado à produção econômica de bens e serviços, entende problema como o resultado indesejado de um processo e acontece quando um item controlado não atinge um nível satisfatório (Werkema, 2012).

A filosofia "Lean Manufacturing" é focada na redução de desperdícios em sistemas produtivos. Quanto menor o desperdício maior a qualidade do processo produtivo, melhorando tempos e custos de produção (Ballé, Jones, Chaize, Fiume, 2019). Segundo Liker (2007), o Lean é composto por 4P's, sendo: processos, pessoas, filosofia ("philosofy") e resolução de problemas ("problem solving") e 14 princípios que são: 1) Decisões visando o longo prazo; 2) Fluxo de processo contínuo; 3) Utilizar o sistema puxado; 4) Nivelar o trabalho; 5) Construir uma cultura de solução de problemas; 6) Padronizar; 7) Usar controles visuais; 8) Utilizar tecnologias confiáveis; 9) Desenvolver líderes que entendam dos processos de trabalho; 10) Desenvolver pessoas que sigam a cultura da empresa; 11) Respeitar rede externa de fornecedores e parceiros; 12) Verificar o problema diretamente na fonte; 13) Tomar decisões por consenso e verificar todas as opões; 14) Melhoria contínua e aprendizagem. O Kaizen (do japonês, mudança para melhor) relaciona-se ao Lean e objetiva a solução de problemas por meio do estudo do processo para melhorar o padrão atual (Queiroz \& Oliveira, 2018).

Algumas das principais metodologias de resolução de problemas utilizadas são o ciclo PDCA, o MASP e o DMAIC. O ciclo PDCA é uma metodologia que possui quatro etapas criadas para o controle e otimização de processos. Ela visa garantir o alcance de metas e as informações obtidas por sua aplicação podem ser usadas como fatores de direcionamento de decisões. A Metodologia de Análise e Solução de Problemas [MASP] utiliza a mesma estrutura analítica do PDCA, com foco de suas etapas para a resolução de problemas. No MASP / PDCA o P-Planejamento faz a identificação e elabora plano de ação para resolução do problema. O D-“Do" executa as ações planejadas. C-Checar verifica se a causa do problema foi eliminada e o A-Agir faz a padronização da solução para prevenir que ela ocorra novamente (Mariani, 2005). Outra metodologia relacionada a ciclos é a DMAIC. Ele é composto pelas etapas D-Definir, M-Medir, A-Analisar, I-Implementar e C-Controlar e muitas vezes é utilizado em conjunto com métodos estatísticos (Nicoletti, 2007). Os métodos PDCA e DMAIC não são conflitantes, mas sim complementares e ambos podem ser usados na mesma organização (Werkema, 2012).

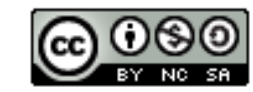



o setor metalmecânico paulista. Brazilian Journal of Production Engineering, 7(5), 30-47 .

O planejamento e a execução de uma metodologia para resolver um problema pode ser compreendido como um projeto, já que é esforço temporário empreendido para criar um resultado exclusivo. $\mathrm{O}$ uso de gestão de projetos é adequado para esse processo de trabalho (Camargo, 2018).

A gestão tradicional de projetos é utilizada quando o escopo e os requisitos são bem definidos no início do projeto. O PMBoK, ISO 21500 e a ICB/IPMA são guias aplicados para esse tipo de gestão. Esses guias convergem principalmente às seguintes áreas do conhecimento: integração, escopo, cronograma, custos, qualidade, recursos, comunicações, riscos, aquisições e partes interessadas. Em termos de processos, os elementos em comum são: inicialização, planejamento, execução, monitoramento e controle e encerramento (Carvalho \& Rabechini Junior, 2018).

A gestão ágil de projetos é aplicada para os casos em que não se tem uma visão clara do escopo, mas sim uma perspectiva do que se pretende desenvolver. Nesse caso, é necessário utilizar ciclos interativos com foco no valor participação do cliente e stakeholders-chave. A gestão ágil foi inicialmente formulada para desenvolvimento "software", mas seus valores e princípios podem ser aplicados para os mais diferentes projetos (Capaldo, 2007).

A gestão híbrida de projetos alia as práticas e conceitos da gestão tradicional com a gestão ágil. Seu objetivo é equilibrar o planejamento e a execução combinando a adaptabilidade da gestão ágil e o controle e parametrização do da gestão tradicional (Menezes, 2018).

Neste trabalho será discutido como os problemas podem ser administrados sob a ótica de gestão de projetos. A hipótese a ser validada é que, em muitos casos, alguns problemas não são tratados como projetos dentro das empresas o que prejudica a sua resolução e gerenciamento. Desse modo, a questão norteadora desta pesquisa é: como as principais metodologias de resolução de problemas podem ser administradas com técnicas de gestão de projetos visando a otimização de processos, e o objetivo que vai atender a está questão de pesquisa está em: identificar na literatura as principais metodologias de resolução de problemas, coletar dados sobre como isso é administrado na indústria metalmecânica na região do estado de São Paulo e propor uma abordagem de gestão de projetos para otimizar tarefas, planejar a gestão de atividades e melhorar processos de resolução de problemas.

\section{Metodologia}

Pesquisas científicas iniciam-se com uma questão e objetivos de pesquisa, que podem resultar de experiências do próprio pesquisador ou de achados na literatura, e trazer contribuições ao campo do conhecimento (Gabriel, 2014). O conhecimento científico diferencia-se de outros conhecimentos por utilizar-se de levantamentos e análises metódicas e sistemáticas, sempre bem estruturadas, e que podem ser verificadas e comprovadas (Raupp \& Beuren, 2008; Gerhardt \& Souza, 2009; Zanella, 2011).

Neste capítulo foram descritos os aspectos metodológicos adotados na pesquisa, quanto à classificação geral, natureza, métodos de pesquisa, objetivos de pesquisa, procedimento técnico, técnica de coleta e análise dos dados.

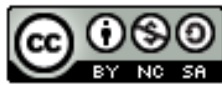



o setor metalmecânico paulista. Brazilian Journal of Production Engineering, 7(5), 30-47 .

A pesquisa conduzida foi de natureza aplicada. Esse tipo de pesquisa visa produzir aplicações práticas para casos concretos. Desse modo, além de produzir conhecimento, a pesquisa gera resultados imediatos em termos econômicos (Fontelles, Simões, Farias, Fontelles, 2009). Neste trabalho, a revisão da literatura sobre resolução de problemas e a análise do setor metalmecânico do estado de São Paulo, permitiram que os conceitos de gerenciamento de projetos fossem aplicados buscando otimização de resultados.

Sob o ponto de vista de seus objetivos é exploratória, bibliográfica, descritiva e prescritiva. A pesquisa exploratória busca proporcionar uma maior compreensão de um tema para torná-lo mais explícito e apresentar proposições. Na maioria dos casos ela envolve um levantamento bibliográfico, coleta e análises de dados. Já a análise descritiva visa descrever determinadas característica de um fenômeno ou população (Gil, 2007). Por fim, a pesquisa prescritiva, propõe soluções que fornecem uma resposta direta ao problema apresentado e estudado (Bonat, 2009).

O levantamento bibliográfico realizado nesta pesquisa ocorreu nas bases de dados (i) Google Acadêmico; (ii) Biblioteca PECEGE e (iii) Biblioteca USP digital, e partiu das palavraschave: (i) gestão de projetos; (ii) resolução de problemas; (iii); a qual possibilitou encontrar uma série de livros e artigos científicos na direção esperada desta pesquisa.

Para coletar os dados, foi utilizado um questionário aplicado pela internet de forma a obter uma pesquisa de opinião pública, com as identidades dos participantes ou das empresas que atuam mantidas anônimas e não identificáveis. O link para a pesquisa foi divulgado em grupos de profissionais da área e o acesso e preenchimento foi feito de forma espontânea. $\mathrm{O}$ foco do questionário visou avaliar exclusivamente a opinião de profissionais sobre temas do seu cotidiano. O método escolhido de coleta de dados foi a escala de Likert. Esse tipo de escala é uma das mais usadas metodologias para aplicação em pesquisas de opiniões, atitudes e sentimentos de forma estruturada e quantitativa. As questões na escala Likert apresentam afirmações auto descritivas e oferecem como opção de resposta uma escala de pontos onde os extremos avaliam se o participante concorda completamente ou discorda completamente (Nemoto \& Beglar, 2014) (Tabela 1).

Tabela 1. Exemplo de escala Likert

\begin{tabular}{ccccc}
\hline \multicolumn{5}{c}{ ESTOU SATISFEITO COM O SERVIÇO QUE RECEBO } \\
\hline $\begin{array}{c}\text { Discordo } \\
\text { totalmente }\end{array}$ & $\begin{array}{c}\text { Discordo } \\
\text { parcialmente }\end{array}$ & $\begin{array}{c}\text { Não concordo } \\
\text { nem discordo }\end{array}$ & $\begin{array}{c}\text { Concordo } \\
\text { parcialmente }\end{array}$ & $\begin{array}{c}\text { Concordo } \\
\text { totalmente }\end{array}$ \\
\hline 1 & 2 & 3 & 4 & 5 \\
\hline
\end{tabular}

Fonte: Stefano, Righi, Lisboa, Godoy (2007)

Esta pesquisa possui abordagem quantitativa. Essa abordagem se caracteriza pelo emprego estatístico tanto da coleta quanto da análise dos dados. Nesse tipo de estudo o pesquisador utiliza um plano preestabelecido com hipóteses e variáveis definidas. O foco é medir e quantificar os resultados da investigação elaborando uma análise estatística (Zanella, 2011). Este trabalho visou compreender as necessidades dos profissionais da área e das empresas que atuam para propor soluções de aprimoramento utilizando gerenciamento de projetos.

Adotou-se o método comparativo. Nessa abordagem devem ser estabelecidas semelhanças e diferenças entre dois ou mais fenômenos com circunstâncias em comum para estabelecer 

o setor metalmecânico paulista. Brazilian Journal of Production Engineering, 7(5), 30-47 .

relações de causas e efeitos (Tiernoa, Gonzalez-Cruz, Martinez, 2017). Os sistemas a serem comparados são os dados coletados na pesquisa de opinião e à literatura de gestão de projetos e resolução de problemas.

O procedimento técnico a ser utilizado é o levantamento. Nesse procedimento são solicitadas informações a um grupo de pessoas sobre um problema a ser estudado, em seguida são feitas análises quantitativas para obter-se conclusões correspondentes aos dados coletados. Ele proporciona informações gerais a respeito de uma população e são indispensáveis em boa parte das investigações sociais. Quando o levantamento não recolhe informações de todos os integrantes de um universo pesquisado é necessário obter-se uma amostra significativa levando em consideração fatores como margem de erro (Gil, 2007). Foi definido que os dados coletados seriam de uma amostra de profissionais da região do estado de São Paulo e que atuam na indústria metalmecânica.

Com os aspectos metodológicos da pesquisa definidos, seguiu-se sistematicamente um protocolo. O desenvolvimento de um protocolo deve conter os procedimentos, instrumentos e regras que devem ser seguidas durante um processo de pesquisa. Seu uso é uma tática para aumentar a confiabilidade da pesquisa, facilitando a coleta de dados (Yin, 2005).

O protocolo proposto nesta pesquisa contém os procedimentos e 8 etapas que foram executadas para coleta e análise dos dados. Seu objetivo é tornar mais claro o desenvolvimento lógico desta pesquisa servindo como guia para sua execução. As etapas do protocolo basearam-se no modelo proposto por Toledo e Shiraishi, (2009) (Tabela 2).

Tabela 2. Protocolo de Pesquisa

\begin{tabular}{|c|c|c|c|}
\hline Capítulo & Etapa & Tópico do Protocolo & Detalhamento \\
\hline Introdução & 1 & Embasamento teórico & Revisão bibliográfica \\
\hline \multirow{12}{*}{ Metodologia } & \multirow{5}{*}{2} & \multirow{5}{*}{$\begin{array}{l}\text { Definição dos aspectos } \\
\text { metodológicos da pesquisa: }\end{array}$} & Natureza \\
\hline & & & Objetivo \\
\hline & & & Abordagem \\
\hline & & & Método \\
\hline & & & Procedimento Técnico \\
\hline & \multirow{2}{*}{3} & \multirow{2}{*}{ Definição do universo de estudo } & Área geográfica \\
\hline & & & Setor \\
\hline & 4 & Fontes de evidência & Definição do perfil de participantes \\
\hline & & & Análise de variáveis \\
\hline & \multirow{3}{*}{5} & \multirow{3}{*}{ Instrumentos de coleta de dados } & Planejamento do questionário \\
\hline & & & Definição de questões \\
\hline & & & Método de aplicação do questionário \\
\hline \multirow{4}{*}{$\begin{array}{l}\text { Resultados e } \\
\text { discussão }\end{array}$} & \multirow{3}{*}{6} & \multirow{3}{*}{$\begin{array}{l}\text { Análise metodológica dos dados } \\
\text { coletados }\end{array}$} & Explicitar as oportunidades de melhoria \\
\hline & & & Comparação com a literatura \\
\hline & & & Discussão dos resultados \\
\hline & 7 & Proposta de melhoria & $\begin{array}{l}\text { Com base na análise dos dados, propor uma } \\
\text { melhoria para o processo }\end{array}$ \\
\hline Conclusão & 8 & Avaliar proposta e concluir & Conclusão final do projeto \\
\hline
\end{tabular}


Citação (APA): Picon, A. B. R , \& Gonçales, M., Filho. (2021). Resolução de problemas e gestão de projetos: proposição de otimização para o setor metalmecânico paulista. Brazilian Journal of Production Engineering, 7(5), 30-47 .

A primeira etapa foi o embasamento teórico por meio da revisão de literatura e está apresentado no capítulo de introdução. A seguir serão delineados os Tópicos do Protocolo da Tabela 1 relacionados à metodologia desta pesquisa.

A segunda etapa é a definição dos aspectos metodológicos. Uma síntese da classificação geral empregada nesta pesquisa, está apresentada na Tabela 3.

Tabela 3. Classificação geral da pesquisa

\begin{tabular}{|c|c|c|c|}
\hline Critério & Classificação & Descrição & Referência \\
\hline Natureza & Aplicada & $\begin{array}{l}\text { Produz aplicações práticas para casos concretos, gera } \\
\text { conhecimento e possibilita resultados imediatos }\end{array}$ & $\begin{array}{l}\text { (Fontelles, } \\
\text { 2009) }\end{array}$ \\
\hline \multirow{4}{*}{ Objetivo } & Exploratória & $\begin{array}{c}\text { Busca proporcionar uma maior compreensão de um } \\
\text { tema para torná-lo mais explícito e apresentar } \\
\text { proposições. }\end{array}$ & \multirow[t]{3}{*}{ (Gil, 2007) } \\
\hline & Bibliográfica & Revisão da Literatura & \\
\hline & Descritiva & $\begin{array}{l}\text { Visa descrever determinadas características de um } \\
\text { fenômeno ou população }\end{array}$ & \\
\hline & Prescritiva & $\begin{array}{c}\text { Propõe soluções que fornecem uma resposta direta ao } \\
\text { problema apresentado e estudado }\end{array}$ & (Bonat, 2009) \\
\hline Abordagem & Quantitativa & $\begin{array}{l}\text { Caracteriza-se pelo emprego estatístico tanto da coleta } \\
\text { quanto da análise dos dados }\end{array}$ & (Zanella, 2011) \\
\hline Método & Comparativa & $\begin{array}{c}\text { Busca relação causal por meio da comparação } \\
\text { sistemática }\end{array}$ & (Tiernoa, 2017 \\
\hline $\begin{array}{l}\text { Procedimento } \\
\text { técnico }\end{array}$ & Levantamento & $\begin{array}{l}\text { São solicitadas informações a um grupo de pessoas e em } \\
\text { seguida são feitas análises quantitativas para obter-se } \\
\text { conclusões correspondentes aos dados coletados }\end{array}$ & (Gil, 2007) \\
\hline
\end{tabular}

Fonte: Autores

A terceira etapa foi a definição do universo de estudo. O local escolhido para a realização desta pesquisa foi o estado de São Paulo. Segundo o Confederação Nacional da Indústria [CNI] (2021) este estado possui Produto Interno Bruto [PIB] industrial de R \$ 391,4 bilhões, equivalente a $29,8 \%$ da indústria nacional e emprega 2.922 .404 trabalhadores na indústria. Em São Paulo estão 25,6\% do total de empresas que atuam no setor industrial do Brasil. O setor metalmecânico foi escolhido devido a experiência do autor dessa pesquisa nessa área e a possibilidade de divulgar a pesquisa em sua rede de contatos. Por fim, o participante, além de atuar em São Paulo e ser do setor metal mecânico, precisa ter alguma experiência, teórica ou profissional, nas áreas de Gestão de Projetos ou Resolução de Problemas. O objetivo deste quesito é importante, pois o questionário é embasado nesse tipo de experiência.

Com o universo definido, a quarta etapa foi analisar as fontes de evidência para o estudo de campo. Devido a limitação do universo de estudo descrito anteriormente, foi preciso criar um método de filtrar se o participante está dentro do universo estipulado. Esse filtro visa garantir fontes de evidências confiáveis. Ao formular o questionário foi definido que seriam feitas questões com a função somente de filtro visando os participantes que tenham a experiência desejada e atuam na área do estudo. Os participantes que não se enquadraram nesses quesitos tiveram as suas respostas desconsideradas nesta pesquisa.

No início do questionário, apresentado no subitem 6.1, foram incluídas as questões que identificaram e desconsideraram parte do potencial de entrevistados, cujas opções de respostas eram somente sim ou não:

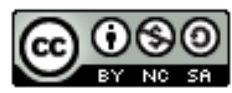



o setor metalmecânico paulista. Brazilian Journal of Production Engineering, 7(5), 30-47 .

- Você atua profissionalmente na região do estado de São Paulo?

- Você atua na indústria metalmecânica (incluindo metalurgia, usinagem, fabricantes máquinas, equipamentos e veículos)?

- Você já utilizou metodologias de resolução de problemas como PDCA, DMAIC, KAIZEN, LEAN, 8D ou outras?

- Possui experiência em Gestão de projetos?

Seguindo o protocolo, foram definidos os instrumentos de coleta de dados referente à quinta etapa. A aplicação do questionário foi feita pelo site "Google Forms" e ficou disponível de 18/02/2021 até 06/04/2021 (https://docs.google.com/forms/d/e/1FAIpQLSfdT51aOxTiSTx899P4jVABpcHo2PISgPlcazzyzsnLlzY6Q/viewform?usp=sf_link).

Ao acessar o link, antes de iniciar a pesquisa, foi apresentado um Termo de Consentimento Livre e Esclarecido. Esse termo visou deixar claro para o participante a garantia de anonimato, o formato da pesquisa, os riscos em participar, os tipos de dados levantados, o objetivo, benefícios da pesquisa, responsáveis, direito de desistir a qualquer momento, o uso da informação e contato do pesquisador. Após o texto uma pergunta com as opções de apenas sim ou não deixavam claro que o participante leu o termo, concordou e aceitou participar da pesquisa. Caso algum participante selecionasse a opção não, sua pesquisa seria desconsiderada.

Com a forma de coleta de dados definida, foi necessário fazer uma análise das variáveis relacionadas a hipótese. Para fins de pesquisa pode-se entender como variáveis, os elementos que podem ser classificados em duas ou mais categorias. É necessário que a hipótese tenha uma relação com as variáveis coletadas e o trabalho precisa ser planejado de modo a verificar o quanto determinadas condições atuam na provável ocorrência do fato (Gil, 2007). No caso desta pesquisa, a hipótese de que, em muitos casos, alguns problemas não tratados como projetos dentro das empresas, o que prejudica à sua resolução e gerenciamento das atividades, tem como variáveis as opiniões de profissionais da área sobre como os problemas são tratados em seu ambiente de trabalho.

Outro ponto importante para a coleta de dados é a análise de erro amostral. O erro amostral pode ser compreendido como como a diferença entre o resultado da amostra e o resultado verdadeiro de uma população. Em muitos casos não é possível obter dados de uma população por fatores como tempo e custo, então é necessária uma amostra que representa essa população. Ao utilizar uma amostra não podemos evitar a ocorrência de um erro amostral, mas podemos limitar seu valor escolhendo uma amostra de tamanho adequado. Isso pode ser feito pelo uso das seguintes fórmulas:

$\mathrm{n}_{0}=\frac{1}{\mathrm{E}_{0}^{2}}$

onde, $\mathrm{n}_{0}$ : primeira aproximação para o tamanho da amostra; e $\mathrm{E}_{0}$ é o erro amostral tolerável

$\mathrm{n}=\frac{\mathrm{N} \cdot \mathrm{n}_{0}}{\mathrm{~N}+\mathrm{n}_{0}}$

onde, n: tamanho da amostra; $\mathrm{N}$ : tamanho da população; e $\mathrm{n}_{0}$ : primeira aproximação para o tamanho da amostra; (Fontelles, Simões, Almeida, Fontelles, 2010) 

o setor metalmecânico paulista. Brazilian Journal of Production Engineering, 7(5), 30-47 .

Nesta pesquisa, devido às limitações de tempo e custo foi considerado um erro amostral de $15 \%$. Como não há dados do número de profissionais da indústria metalmecânica de São Paulo, considerou-se os dados do CNI de que atualmente 2.922.404 de pessoas atuam na indústria brasileira sendo 25,6\% em São Paulo. Logo o universo de estudo está contido nesse número e é de 730.601 pessoas. Aplicando esses dados nas equações (1) e (2) temos

$\mathrm{N}=730.601$ pessoas

$E_{0}=0,15$

$\mathrm{n}_{0}=44,4$ pessoas

$\mathrm{n}=44,39$ pessoas

Logo a meta foi obter questionários válidos de pelo menos 45 pessoas considerando o erro amostral proposto.

Após o planejamento minucioso e sistematizado para a realização desta pesquisa, iniciou-se a coleta de dados apresentada no capítulo de resultados e discussão.

\section{ReSUltados E DiscuSSÃo}

A coleta dos dados permitiu iniciar a análise exploratória para tornar o tema mais explícito de forma a compreender o fenômeno. A coleta de dados visou estudar a hipótese de que, em muitos casos, alguns problemas não tratados como projetos dentro das empresas prejudica à sua resolução e gerenciamento.

No total foi possível coletar 81 respostas. O critério de filtro como atuar profissionalmente na região do Estado de São Paulo, atuar na indústria metalmecânica e ter experiência em Gestão de Projetos ou metodologias de resolução de problemas foram aplicados e reduziram o número de respostas válidas para 48 (59\% dos participantes). Sendo $41 \%$ das respostas desconsideradas por não atenderem aos requisitos do universo de estudo. $\mathrm{O}$ número de respostas válidas foi superior à meta para um erro amostral de $15 \%$. Uma visão do resultado pode ser observada na Figura 1.

Figura 1. Participantes da Pesquisa

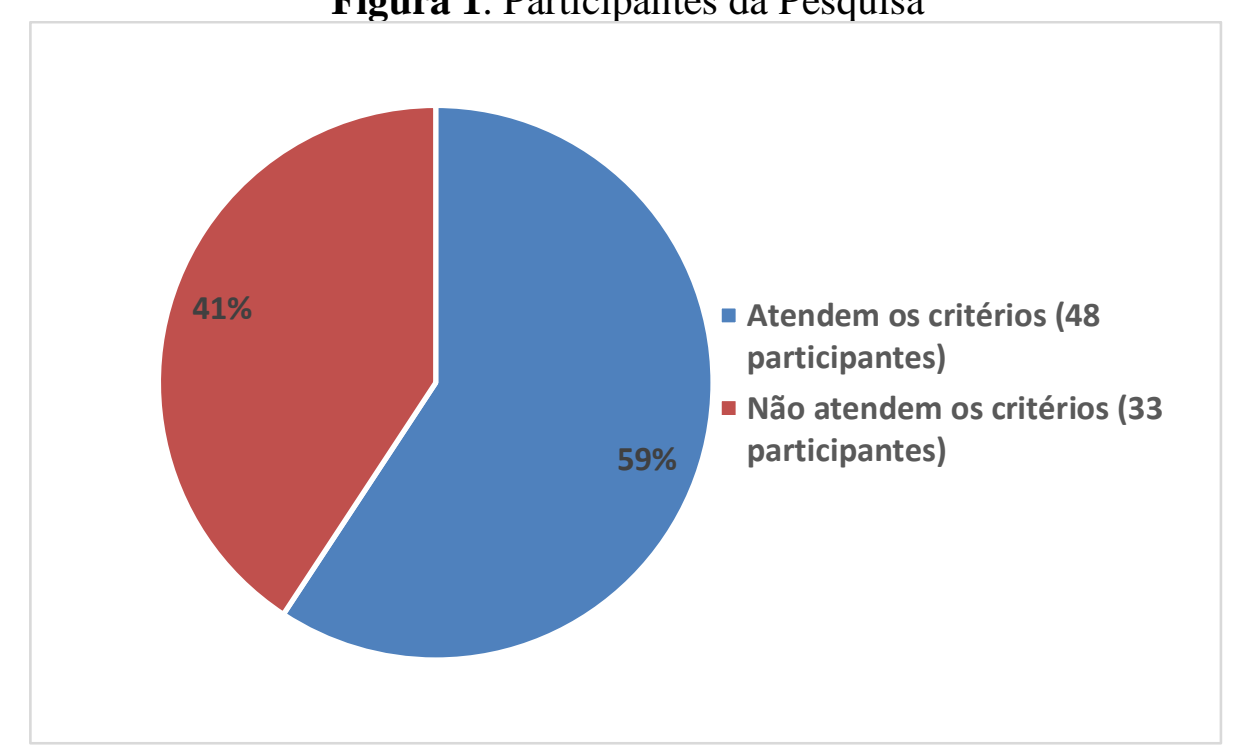

Fonte: Autores

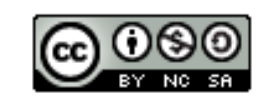



o setor metalmecânico paulista. Brazilian Journal of Production Engineering, 7(5), 30-47 .

\subsection{Análise das respostas.}

A análise metodológica dos dados coletados, referente à sexta etapa do protocolo, foi feita de forma estatística com o auxílio do software Minitab. A questão 1 foi "Onde eu trabalho os problemas sempre são tratados como projetos? A questão objetivou avaliar a complexidade, definiu-se a metodologia de resolução e tem-se a visibilidade de características como prazo, custo, escopo, riscos, etc." A Figura 2 apresenta a análise estatística das respostas desta questão.

Figura 2. Análise da questão 1

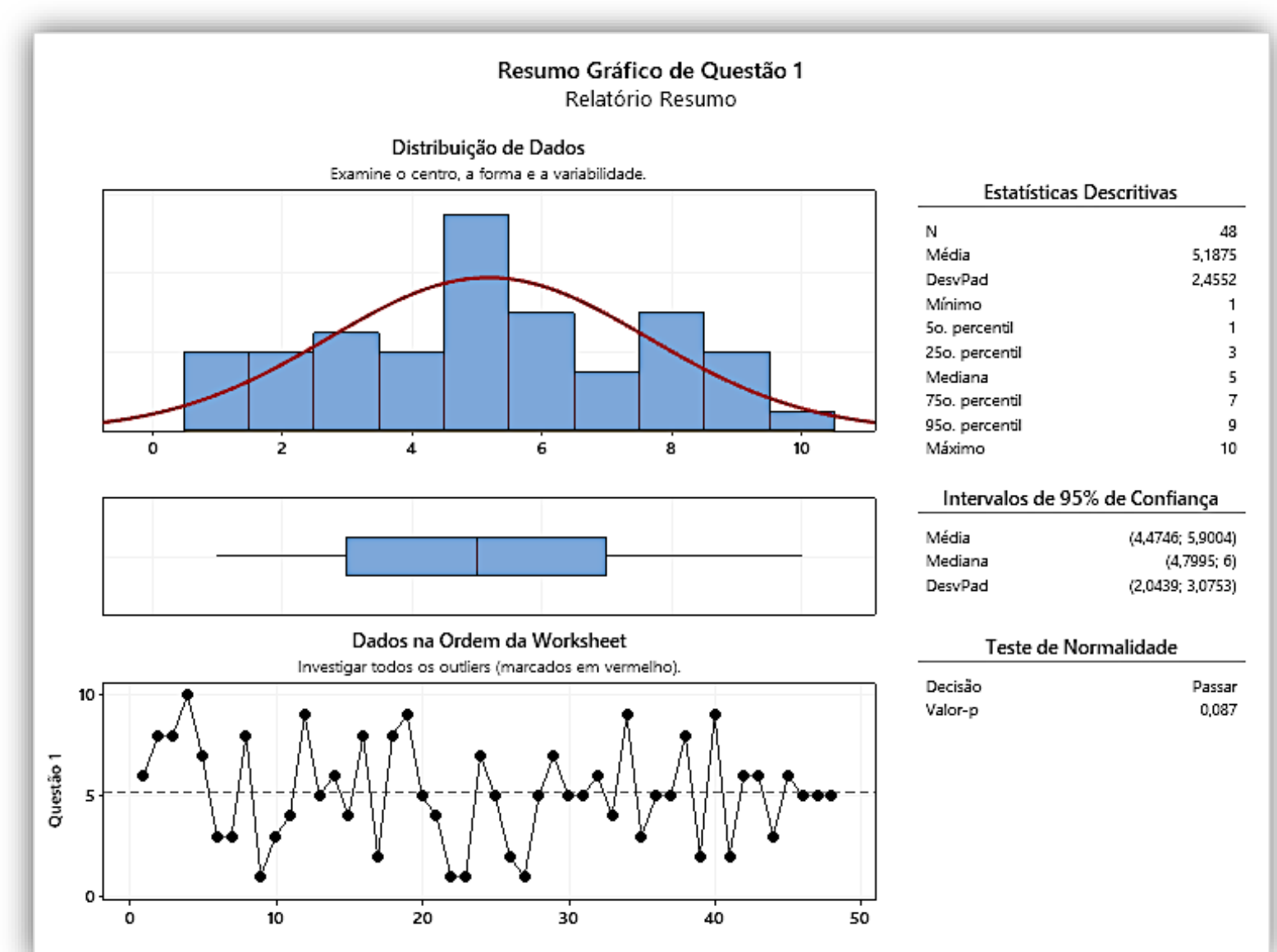

Fonte: Autores

Identificou-se que nesse caso os dados tenderam ao centro da escala, com média de 5,1875 e desvio padrão de 2,4552. O teste do Valor-P apresentou resultado de $0,087(>0,05)$, logo os dados seguem uma distribuição normal, ou seja, em uma amostra maior a distribuição deve se manter em uma curva semelhante. A conclusão dessa análise gráfica é que a maioria das empresas tendem a ter um tratamento neutro de problemas como projetos, ou seja, em alguns casos as empresas tratam como projetos e em outros não. A quantidade menor nas extremidades mostra que em poucos casos todos os problemas são tratados como projetos ou que nenhum problema é tratado como tal.

A questão 2 foi: Quando acontece um problema as pessoas envolvidas sempre fazem um planejamento prévio antes de iniciar às ações de resolução? A Figura 3 apresenta o estudo quantitativo das respostas da questão 2 .

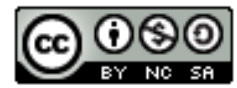



o setor metalmecânico paulista. Brazilian Journal of Production Engineering, 7(5), 30-47 .

Figura 3. Análise da questão 2

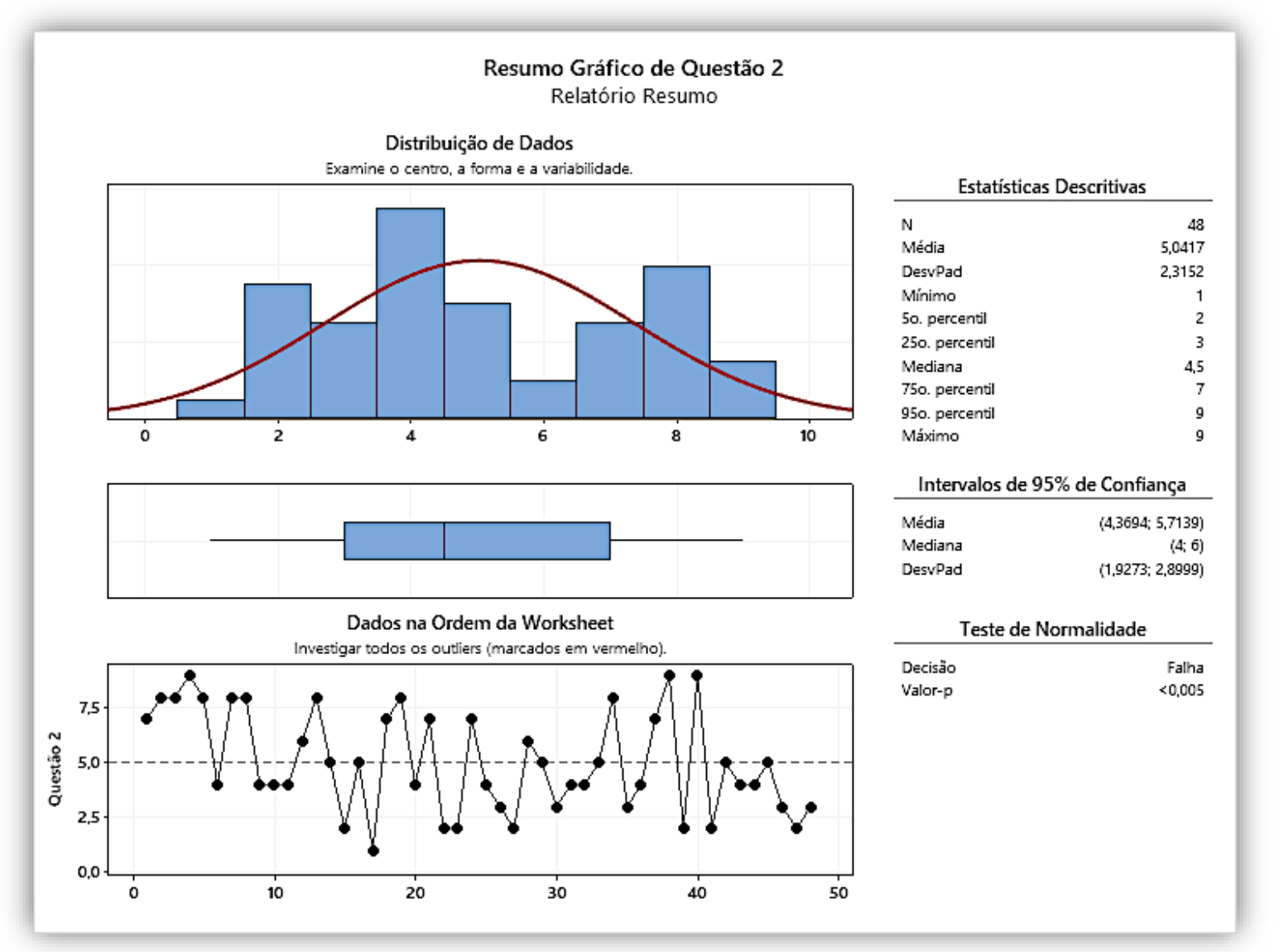

Fonte: Autores

Nesse caso identifica-se que a média de 5,0417 e desvio padrão 2,3152 indicam que a maioria dos dados ficou na faixa central do gráfico. O teste do Valor-P para normalidade foi $<0,005$, ou seja, a curva não ser normal. Pela dispersão dos dados, pode-se afirmar que, na maioria dos casos, a resposta é neutra. Isso indica que o planejamento prévio para resolver um problema em alguns casos é feito e em outros não. O extremo superior indica que em poucos casos sempre é feito um planejamento e o inferior que em poucos casos nunca é feito nenhum tipo de planejamento.

A questão 3 foi: Onde eu trabalho há uma forma clara de determinar qual metodologia de resolução de problemas será utilizada levando em consideração fatores como custo, prazo e complexidade? A Figura 4 apresenta a distribuição de dados e análise estatística da questão 3 mostrando uma tendência ao centro da escala. 

o setor metalmecânico paulista. Brazilian Journal of Production Engineering, 7(5), 30-47 .

Figura 4. Análise da questão 3

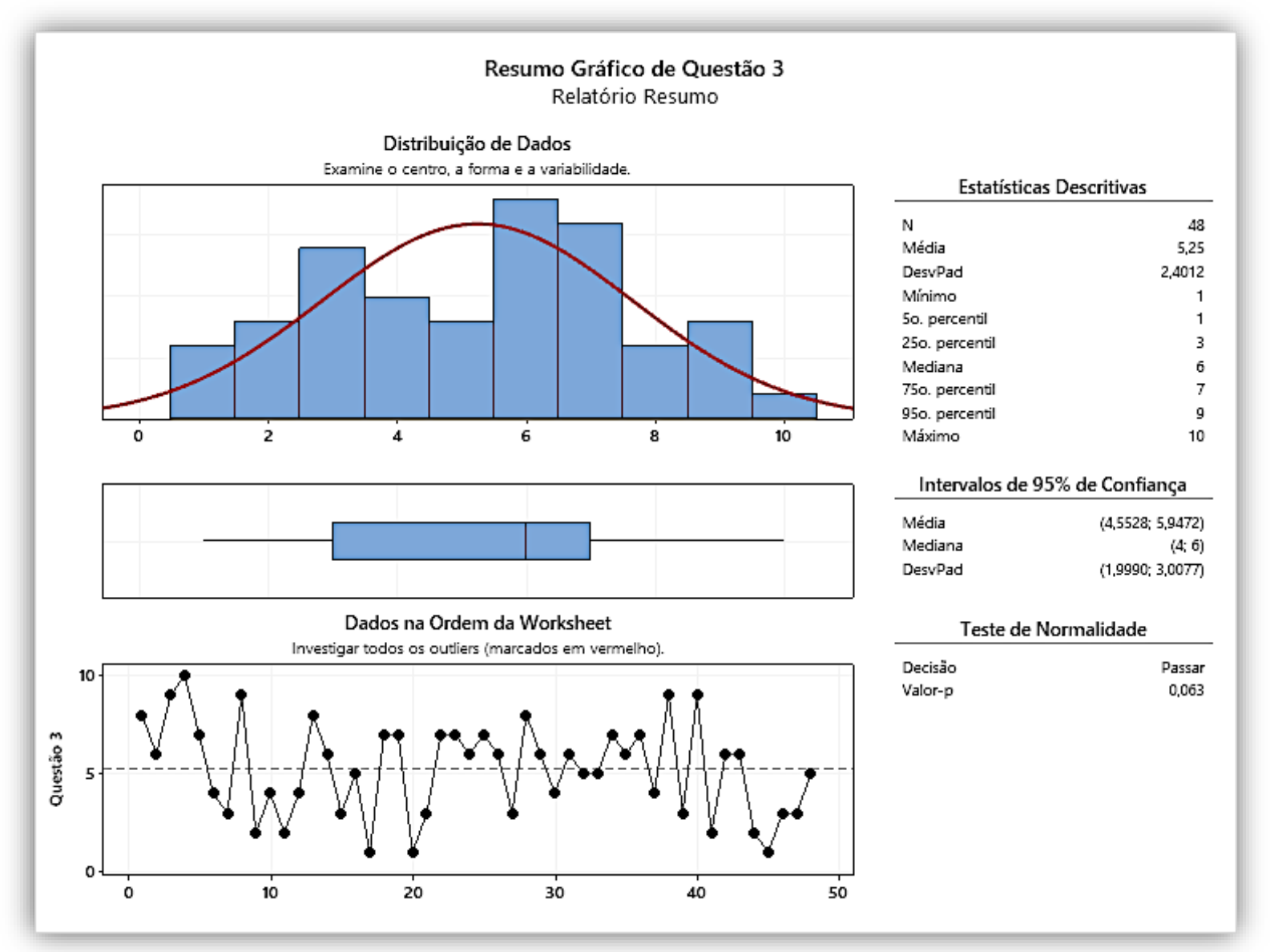

Fonte: Autores

Novamente as respostas tenderam ao centro. A média de 5,4012 e desvio padrão de 2,4012 teve distribuição normal com Valor-P de 0,063. Há um leve deslocamento para a direita com a mediana em 6. Pode-se afirmar que a questão da escolha da metodologia de resolução de problemas também é neutra, ou seja, em alguns casos há uma forma de determinar à metodologia e outros não.

A questão 4: Antes de iniciar a resolução de um problema eu considero importante avaliar a complexidade para então de definir o esforço para documentar e gerir a resolução? e a questão 5: Acredito que seria interessante uma ferramenta ou método para analisar a complexidade de um problema, definir a metodologia de resolução, planejar a documentação e definir as técnicas de gerenciamento das atividades? Tiveram um grande deslocamento para a direita (alto índice de pessoas que concordam com as afirmações). Por serem curvas deslocadas não foi feito teste de normalidade. Pode-se concluir pelos gráficos que a maioria dos profissionais concordam que é preciso avaliar a complexidade antes de definir o esforço para gerir e documentar a solução e que a maioria concorda que uma ferramenta para análise de metodologia de resolução, definir técnicas de gerenciamento e analisar a complexidade de um problema é relevante, mais detalhes são apresentados nas Figuras 5 e 6. 

o setor metalmecânico paulista. Brazilian Journal of Production Engineering, 7(5), 30-47 .

Figura 5. Análise da questão 4

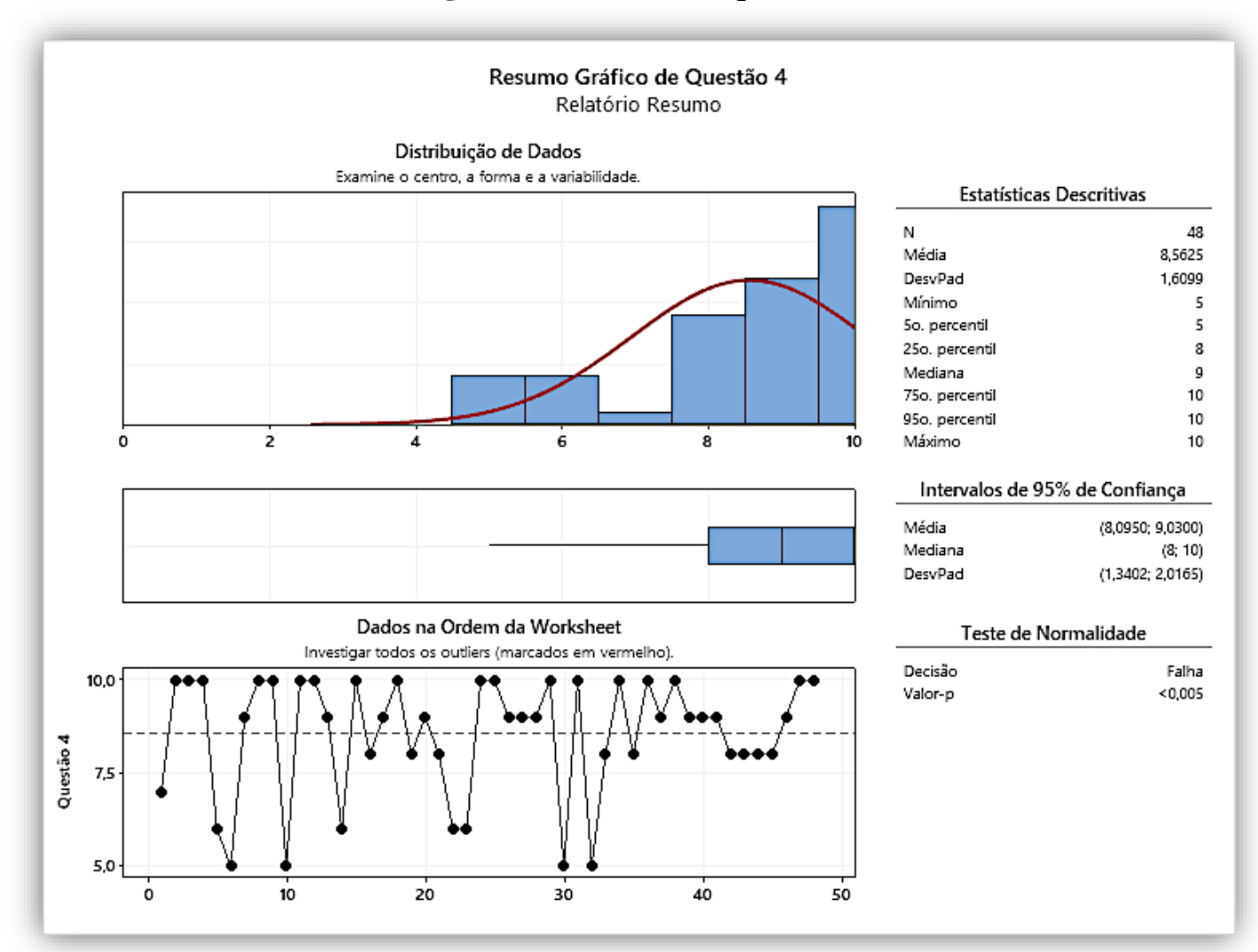

Fonte: Autores

Figura 6. Análise da questão 5

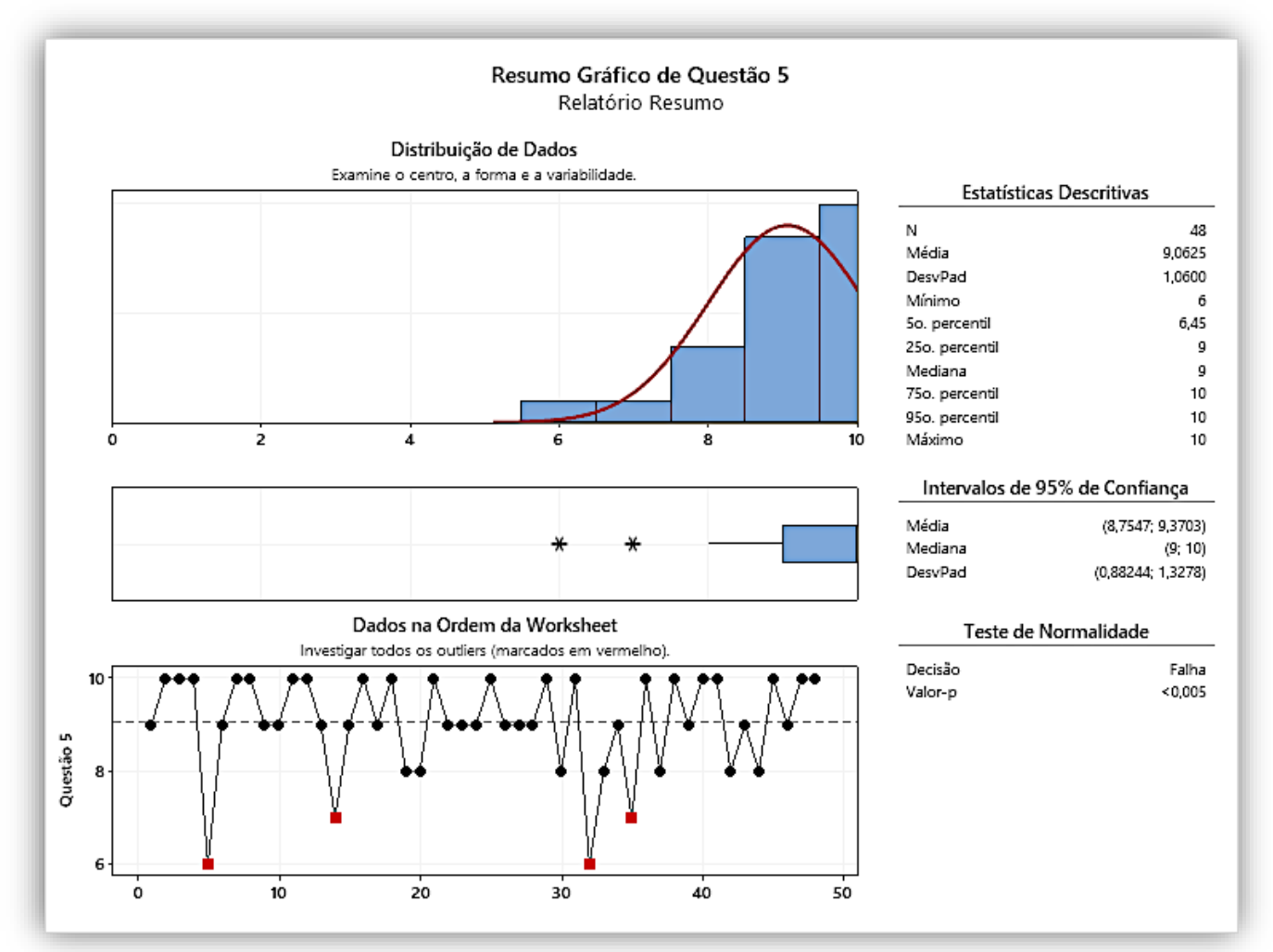

Fonte: Autores 

o setor metalmecânico paulista. Brazilian Journal of Production Engineering, 7(5), 30-47 .

\subsection{Proposição de melhoria}

Com os dados analisados, foi possível executar a sétima etapa do protocolo que é a proposta de melhoria, o resumo da análise dos gráficos foi que claramente não há uma forma padrão na indústria de como os problemas devem ser tratados. Em muitos casos os profissionais não têm visibilidade de fatores importantes como prazo, custo e escopo. Também é possível observar que muitas vezes um planejamento mais detalhado não é feito. Essa diferença de tratamento frente aos problemas nas empresas pode ser explicada pelo nível de maturidade e aplicação do sistema "Lean Manufacturing" nas indústrias que os profissionais atuam. Conforme relatado na introdução desta pesquisa, o Sistema Lean é composto por 14 princípios que são mostrados e formam a base para à proposição de melhoria para o estudo.

Para à proposição de melhoria segundo os 14 princípios, foi preciso uma metodologia para garantir que alguns pontos fossem avaliados, como:

- Construir uma cultura de solução de problemas: A metodologia deve promover discussão e participação de todo o time envolvido no problema;

- Criar fluxo de processo contínuo: A metodologia precisa ser utilizada para todos os casos e não somente em situações pontuais;

- Padronizar: A forma de estudo precisa ser padronizada

- Verificar o problema diretamente na fonte: A metodologia deve promover uma análise que busque a causa raiz e faça o time atuar fisicamente na fonte do problema;

- Usar controles visuais: Precisa ser simples e visual, controles muito complexos podem desmotivar sua execução;

- Tomar decisões por consenso e verificar todas as opões: Deve promover envolvimento do time

- Desenvolver pessoas que sigam a cultura da empresa: Deve valorizar os bons profissionais que executam a cultura da empresa nas práticas de resolução de problemas;

- Melhoria contínua e aprendizagem: A metodologia deve apontar lições aprendidas.

A proposição e a real contribuição desta pesquisa, embasada pela literatura e pesquisa de campo, consistem em realizar uma reunião ou momento de Planejamento de Solução de Problemas. A reunião não deve passar de 15 minutos para que seja rápida e não afete a produtividade ou motivação do time envolvido. Durante a reunião o responsável pela área onde o problema acontece preenche o quadro "Canvas" apresentado pela Figura 7 em conjunto com o time de trabalho. 

o setor metalmecânico paulista. Brazilian Journal of Production Engineering, 7(5), 30-47 .

Figura 7. Quadro de Planejamento de Solução de Problemas

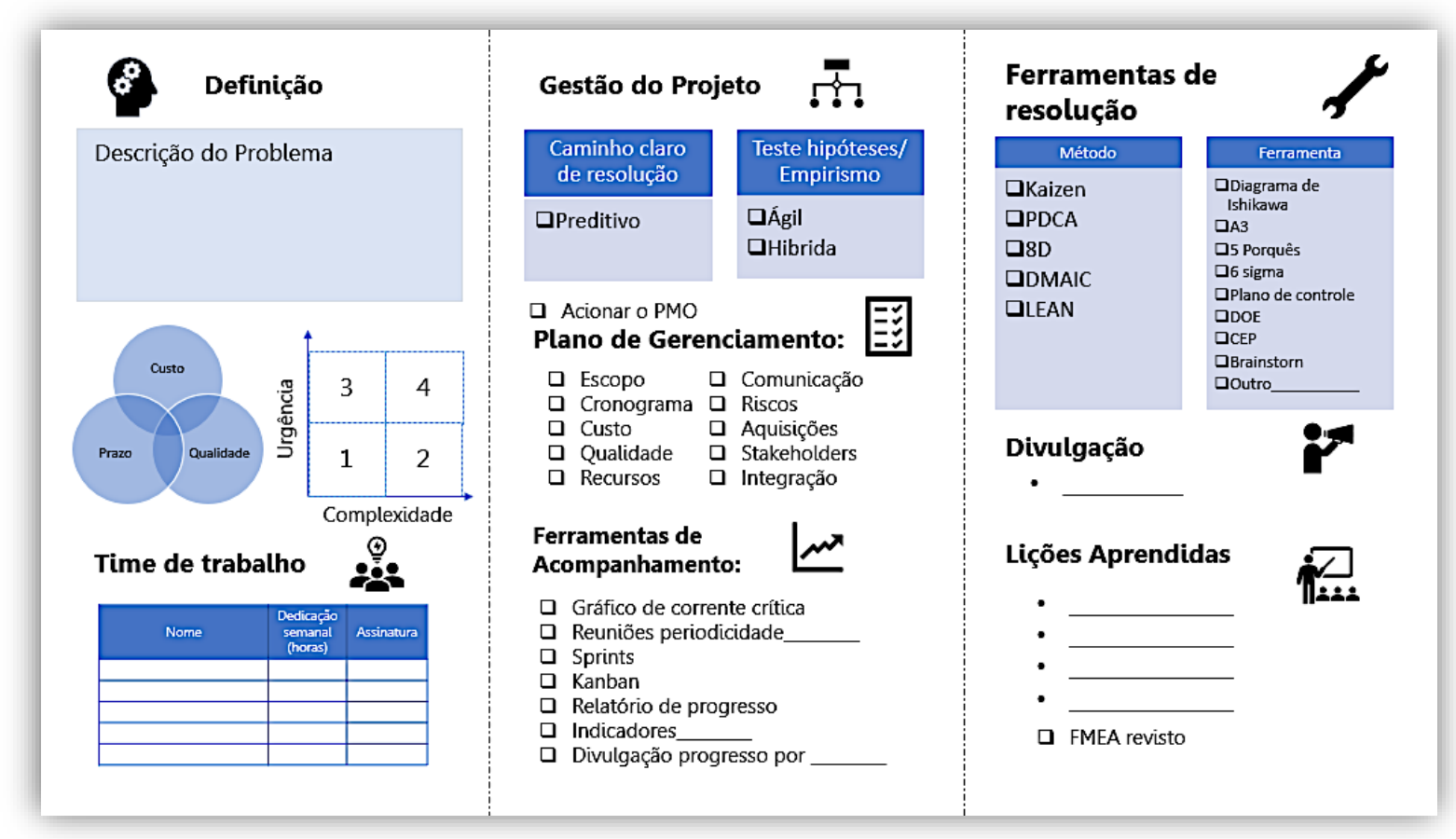

Fonte: Autores

Esse quadro é um controle visual e a sugestão é que ele seja impresso em A3 e o ideal é preenchimento "a mão" para evitar desperdício de tempo com formatação.

Esse quadro tem três grandes frentes de discussão: Definição do Problema, Gestão do Projeto e Ferramentas de resolução. A Definição de Problema (Figura 8) o profissional que apresenta o problema descreve-o para toda a equipe da reunião. Uma segunda pessoa deve anotar a descrição para que seja garantido que o problema foi entendido pela equipe. Após essas atividades o time terá uma base de discussão para classificar o problema como de Custo, Prazo ou Qualidade. Todavia, a classificações de urgência e complexidade podem variar de empresa para empresa, mas é importante definir uma forma padrão para que em diferentes situações a gestão visual seja efetiva. A etapa seguinte é a definição do time envolvido na resolução, dedicação em horas e assinatura para promover o comprometimento, caso a quantidade de horas dedicada seja muito elevada o profissional pode utilizar esse quadro para justificar a necessidade com sua gestão.

Partindo para a etapa de Gestão do Projeto (Figura 9) chega o momento de definir como a solução será gerenciada. Se às etapas de resolução são claras, a sugestão é seguir pelo gerenciamento preditivo. Caso necessite de teste de hipóteses de resolução ou utilização com características empíricas, ágil ou híbrido. Nessa discussão, caso o problema seja muito complexo, o "Project management Office" [PMO] deve ser envolvido para executar uma gestão dedicada ao caso. O plano de gerenciamento é uma forma de discutir e se comprometer com o esforço da documentação. Todos os itens marcados no plano de gerenciamento devem ter sua documentação realizada e entregue antes do encerramento do problema. Por fim é discutido como será feito o acompanhamento das atividades.

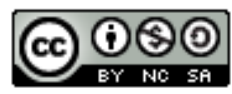



o setor metalmecânico paulista. Brazilian Journal of Production Engineering, 7(5), 30-47 .

Figura 8. Detalhe da etapa de definição e Figura 9. Gestão do Problema como Projeto

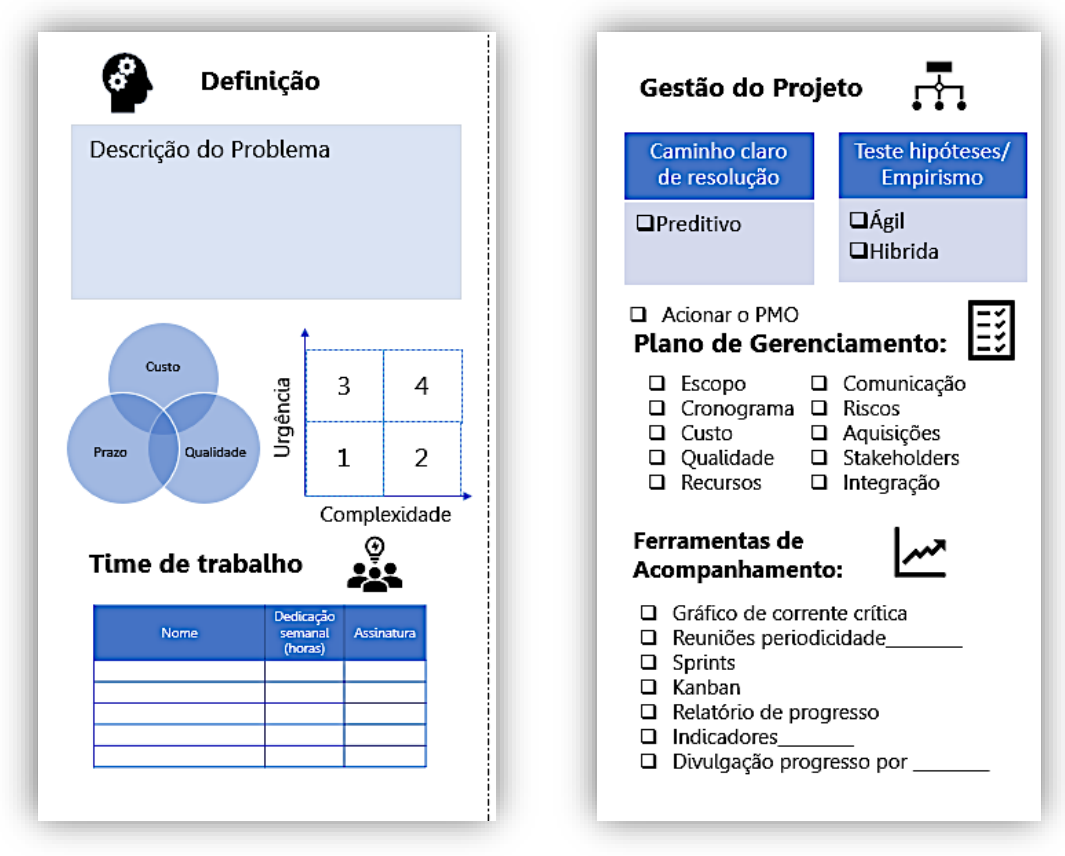

Fonte: Autores

A última etapa da reunião é definir qual metodologia ou ferramentas serão utilizadas na solução. Isso pode depender da experiência dos envolvidos, da prática da empresa ou de fatores que serão discutidos durante a reunião. A Figura 10 apresenta as ferramentas de solução.

Figura 10. Ferramentas de resolução

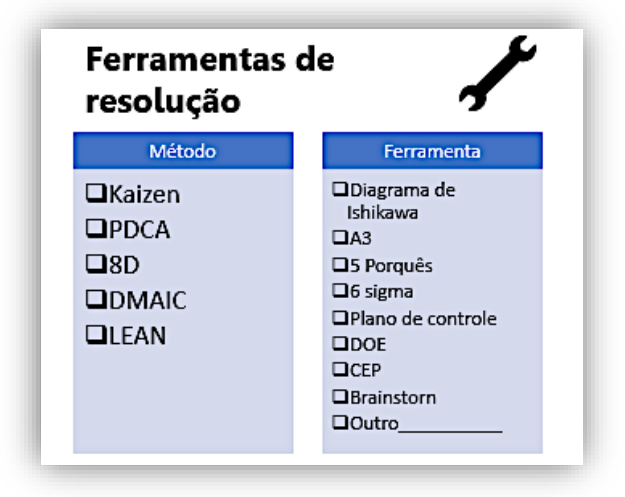

Fonte: Autores

Após a solução do problema o time deve voltar a se reunião para o preenchimento final do quadro conforme Figura 11. Nesse momento é discutido como a divulgação será realizada para promover visibilidade e reconhecimento ao time envolvido. Outro ponto importante é discutir quais foram as lições aprendidas, cumprindo com os princípios da metodologia "Lean", e se houver Análise de Modo e Efeito de Falha [FMEA] é preciso revisitar para identificar a prevenção falhou.

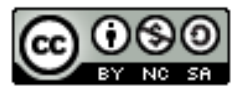



o setor metalmecânico paulista. Brazilian Journal of Production Engineering, 7(5), 30-47 .

Figura 11. Ferramentas de resolução

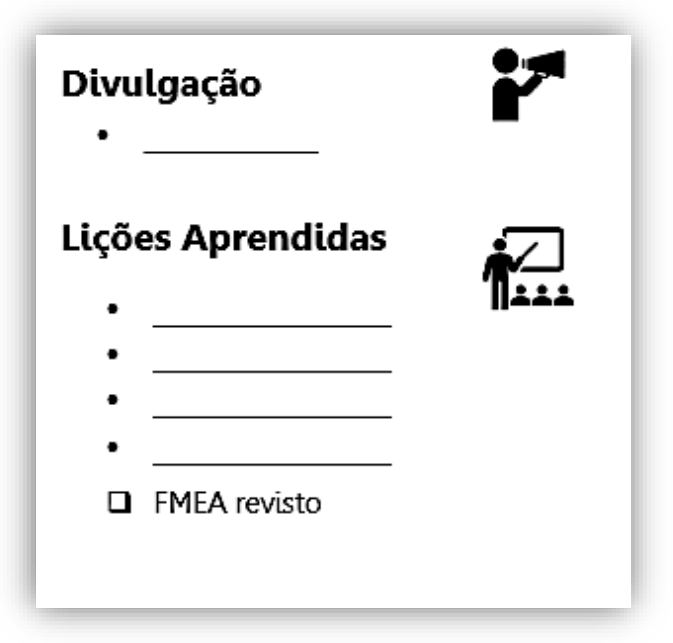

Fonte: Autores

\section{Conclusão}

Este trabalho permitiu que fosse feita uma análise de como os problemas são tratados na indústria metalmecânica do estado de São Paulo. A hipótese de que em muitos casos os problemas não são tratados como projetos pelas empresas e que isso prejudica à sua resolução e gerenciamento foi validada pela pesquisa de opinião aplicada. Foi identificado que em muitos casos não há uma forma clara de como tratar os problemas, que muitas vezes não há um planejamento antes de iniciar as atividades de implementação e nem uma definição de metodologia padrão para resolução dentro das empresas. Outro ponto identificado é que o esforço para resolver um problema deve estar alinhado à sua complexidade e uma ferramenta para ajudar nessa avaliação seria relevante no dia-a-dia. Com base na pesquisa de campo e na revisão da literatura, foi possível realizar uma proposição de solução visando a otimização de processos. Nesse contexto, foi proposto que às empresas considerem um momento ou reunião de Planejamento de Solução de Problemas com etapas definidas visando a aplicação da filosofia "Lean Manufacturing". Todavia, para guiar a aplicação da proposição criou-se um quadro "Canvas" como guia para planejamento da resolução. Para tanto, como sugestão de trabalhos futuros indica-se a possibilidade de testar em campo a aplicação da proposição e analisar suas vantagens e desvantagens.

\section{Referências}

Associação IPMA Brasil de Gerenciamento de Projetos. (2012). National Competence Baseline-NCB. Referencial Brasileiro de Competências. Recuperado de https://www.academia.edu/6606190/Associa\%C3\%A7\%C3\%A3o_IPMA_Brasil_de_Gerenci amento_de_Projetos_NCB_National_Competence_Baseline

Ballé, M., Jones, D., Chaize, J., \& Fiume O. (2019). A Estratégia Lean: Para Criar Vantagem Competitiva, Inovar e Produzir com Crescimento Sustentável (1a ed.). Porto Alegre: Bookman.

Bonat, D. (2009). Metodologia de Pesquisa (3ed)., Curitiba: IESDE Brasil S.A Camargo, M. (2018). Gerenciamento de Projetos (2a ed.). Rio de Janeiro: Grupo GEN. Capaldo, A. D. (2007). Gerenciamento ágil de projetos - Aplicação em produtos inovadores (1a ed.). São Paulo: Editora Saraiva. 
Citação (APA): Picon, A. B. R , \& Gonçales, M., Filho. (2021). Resolução de problemas e gestão de projetos: proposição de otimização para o setor metalmecânico paulista. Brazilian Journal of Production Engineering, 7(5), 30-47 .

Carvalho, M. M., \& Rabechini, R. J. (2018). Fundamentos em Gestão de Projetos Construindo Competências para Gerenciar Projetos (5a ed.). Rio de Janeiro: Atlas.

Confederação Nacional da Indústria [CNI]. 2021. Relatório Estado de São Paulo. Recuperado de https://perfildaindustria.portaldaindustria.com.br/estado/sp

Fontelles, M. J., Simões, M. G., Farias, S. H., \& Fontelles, R. G. S. (2009). Metodologia da Pesquisa Científica: diretrizes para a elaboração de um protocolo de pesquisa. Belém: Núcleo de Bioestatística Aplicado à Pesquisa da Universidade da Amazônia.

Fontelles, M. J., Simões, M. G., Almeida J. C., \& Fontelles R. G. S. (2010). Metodologia da pesquisa: diretrizes para o cálculo do tamanho da amostra. Revista Pan-americana de Medicina, 24, 57-64.

Gabriel, M. L. D. (2014). Métodos Quantitativos em Ciências Sociais: Sugestões para Elaboração do Relatório de Pesquisa. Desenvolvimento em questão, V12 (28): 348-369.

Gerhardt, T. E., \& Souza, A. C. (2009). Métodos de Pesquisa. Série Educação a Distância. (1a ed.). Porto Alegre: Editora da UFRGS.

Gil, A. C. (2007). Como elaborar projetos de pesquisa. (6a ed.). Rio de Janeiro: Atlas.

Günther, H. (2006). Pesquisa qualitativa versus pesquisa quantitativa: esta é a questão? Psic.: Teor. e Pesq, 22(2), 201-209.

Liker, J. (2007). O modelo Toyota: 14 princípios de gestão do maior fabricante do mundo. (1a ed.). Porto Alegre: Bookman Edtora .

Mariani, C. A. (2005). Método PDCA e Ferramentas da qualidade no gerenciamento de processos industriais: Um estudo de caso.RAI - Revista de Administração e Inovação, 2, 110126.

Menezes, L. C. D. M. (2018). Gestão de Projetos. (4a ed.). São Paulo: Atlas.

Nemoto, T., \& Beglar, D. (2014). Developing Likert-Scale Questionnaires. JALT2013 Conference Proceedings, Tokyo, Japon.

Queiroz, A., \& Oliveira, L. (2018). A Ferramenta Kaizen na Solução de Problemas Em Uma Indústria Automobilística. Revista de Engenharia e Pesquisa Aplicada, 3: 128-140.

Raupp, F. M., \& Beuren, I. M. (2008) Metodologia da Pesquisa Aplicável às Ciências Sociais. (3a ed.) São Paulo: Atlas.

Stefano, N., Righi, A. W., Lisboa, M. G., \& Godoy, L. P. (2007). Utilização das dimensões da qualidade e escala Likert para medir a satisfação dos clientes de uma empresa prestadora de serviços. XXVII Encontro Nacional de Engenharia de Produção. Foz do Iguaçu, Brasil.

Tiernoa, N. R., Gonzalez-Cruz, T. F., Martinez, J. L. (2017). An overview of qualitative comparative analysis: a bibliometric analysis. J Innov Knowl, 2(1),15-23.

Toledo, L. A., \& Shiraishi, G. D. F. (2009). Estudo de caso em pesquisas exploratórias qualitativas: Um ensaio para a proposta de protocolo do estudo de caso. Revista da FAE, 12(1), 103-119.

Werkema, C. (2012). Métodos PDCA e Demaic e Suas Ferramentas Analíticas. (1a ed.). Rio de Janeiro: Grupo GEN.

Yin, R. K. (2005). Estudo de caso: planejamento e métodos. (3a ed). Porto Alegre: Bookman.

Zanella, L. C. H. (2011). Metodologia de Pesquisa. (2a ed.). Florianópolis: Departamento de Ciências da Administração/Universidade Federal de Santa Catarina [UFSC]. 